\title{
Nuclear localization of calcium-activated BK channels in skate ampullary electroreceptors
}

\begin{abstract}
Abby L. Chen*, Ting-Hsuan Wu*, Lingfang Shi, William T. Clusin and Peter N. Kao
Division of Pulmonary, Allergy and Critical Care Medicine, Stanford University

School of Medicine, Stanford, CA 94305
\end{abstract}

\section{* Co-first authors}

\section{Abstract}

Ampullae of Lorenzini are sensory organs capable of detecting microvolt gradients in seawater. Electroreception involves interplay between voltage-dependent calcium channels Cav1.3 and big conductance calcium-activated potassium (BK) channels in apical membranes of receptor cells. Expression of BK (kcnma1) and Cav1.3 (cacna1d) channels in skate (Leucoraja erinacea) ampullary electroreceptors was studied by in situ confocal microscopy. BK and Cav1.3 channels colocalize in plasma membranes, ribbon synapses and kinocilia. BK channels additionally colocalize with chromatin and nuclear lamins in electroreceptor cells. Bioinformatic sequence analysis identified an alternatively spliced bipartite nuclear localization sequence (NLS) in kcnma1 (at site of mammalian STREX exon). Skate kcnma1 wild type cDNA transfected into HEK293 cells localized to the endoplasmic reticulum and nucleus. Mutations in the NLS (KR $\rightarrow$ AA or SVLS $\rightarrow$ AVLA) independently attenuated nuclear translocation from endoplasmic reticulum. BK channel localization may be controlled by splicing or phosphorylation to tune electroreception and modulate gene expression. 


\section{Introduction}

Calcium-activated potassium channels were first discovered in the early 1970's and are present in virtually all living organisms. The physiological roles ascribed to these channels have greatly expanded since the well-known review article by Meech in 1978 [1]. Dysfunction of calcium-activated potassium channels are involved in a broad range of neurologic, cardiac and autoimmune diseases, including epilepsy [2], alcoholism [3], cardiac arrhythmias [4, 5], rheumatoid arthritis [6-8] and skeletal myoblast differentiation [9].

Ampullae of Lorenzini are electroreceptive sensory organs capable of detecting nanovolt gradients in seawater $[10,11]$. Canals filled with highly electroconductive keratan sulfate jelly [12] originate anterior to gill slits and terminate in ampullary outpouchings of epithelia, from which the sensory nerve derives. Each ampulla opens into seven alveoli consisting of epithelial sheets of conical electroreceptor cells with apical kinocilia facing the lumen, intercalated between supporting cells that form electrically tight junctions to the receptor cells $[13,14]$.

The types and location of ion channels in the receptor cells was inferred from anatomical, physiological and pharmacologic data [15-20]. In response to an excitatory electrical stimulus (when the lumen becomes more positive), opening of voltagedependent calcium channels produces a depolarizing inward calcium current entering the receptor cell through the apical membrane, that flows outward across the basal membranes, triggering calcium-dependent vesicle release. The excitatory response is terminated by opening of calcium-activated potassium channels in the apical membrane, which repolarizes both faces [21-25]. Spontaneous activity is characterized 
by $20 \mathrm{~Hz}$ oscillations that can be recorded experimentally by placing the ampulla in an air gap $[18,22]$. Voltage-gated potassium channels in the receptor cells contribute to the oscillatory responses $[19,20]$. These oscillatory signals control release of neurotransmitter inferred to be glutamic acid by RNA-ISH localization of SIc17a8 (glutamate transporter 3) [26].

Release of calcium from intracellular stores can also lead to opening of calciumactivated potassium channels. There are two principal types of calcium-activated potassium channels. Small conductance calcium activated potassium channels (SK) have a single channel conductance of 9-14 pS and large conductance channels (BK), have a conductance of $250-300 \mathrm{pS}[27,28]$. BK channels are generally sensitive to both intracellular calcium and membrane voltage, while SK channels are only sensitive to calcium.

Recent studies by Bellono et al. determined the amino acid sequences for Cav1.3 cacna1d and BK kcnma1 channels in skate electroreceptors [29], suggesting that a slight sequence variation in the calcium channel in the skate makes it unusually sensitive to voltage. Relative expression of mRNA transcripts for BK channels was 35fold higher than for SK channels. Furthermore, in situ hybridizations demonstrated high expression of mRNA transcripts for cacna1d and kcnma1 in electroreceptor cells of skate ampulla.

Trafficking of potassium channels to intracellular organelles or plasma membranes is subject to complex regulation that includes alternative splicing to incorporate specific addresses, posttranslational modifications such as phosphorylation or glycosylation and association with accessory subunits [30, 31]. The voltage-gated $\mathrm{K}$ 
channel, Kv10.1 is linked to diverse cancers [32]. Functional Kv10.1 channels localized to the inner nuclear membrane together with Lamin $A / C$ subsequent to exposure to the extracellular milieu [33].

BK channels are regulated by complex alternative splicing, post-translational modifications and protein-protein interactions that together influence subcellular localization and function [27, 34-44]. Alternative splicing at the C-terminus that incorporates amino acids -DEC targets BK channels to mitochondria [45]. Complex splicing at site 4, also known as c2, where the mammalian STREX exon may insert, was shown to affect $\mathrm{BK} \alpha$ subunit channel tissue distribution, trafficking and regulation $[36,38]$. Random insertion of GFP variants into BK channels resulted in generation of 55 unique fluorescent fusion proteins, of which 19 were expressed and functional at the plasma membrane and 36 showed intracellular expression without suggestion of misfolding [46].

Nuclear localization of calcium-activated BK channels has been described [4750]. Li et al [51] demonstrated BK channel expression in hippocampal neurons in a pattern of intracellular rings surrounding nuclei and colocalizing with the nuclear envelope marker lamin B1. Confocal microscopy was used to demonstrate colocalization of BK channels with lamin B1 in inner nuclear membranes. These signals were absent in neurons from mice with targeted knockout of BKa.

Skate ampullae are small and translucent making them suitable for whole mount immunostaining and in situ confocal microscopy. We studied skate electroreceptor expression of voltage-gated calcium channel Cav1.3 and calcium-activated potassium 
bioRxiv preprint doi: https://doi.org/10.1101/2020.01.27.922161; this version posted January 28, 2020. The copyright holder for this preprint (which was not certified by peer review) is the author/funder. All rights reserved. No reuse allowed without permission.

channel BK using antibodies generated against conserved epitopes. Unexpectedly, we discovered prominent nuclear expression of BK channels in electroreceptor cells. 


\section{Results}

Skate ampullary electroreceptors are thin walled epithelial structures that transduce microvolt electrical gradients into membrane oscillations that regulate synaptic transmission. Ampulla with attached canals and sensory nerves were dissected from little skates, Leucoraja erinacea. Freshly dissected ampulla were fixed in paraformaldehyde, permeabilized, and used for whole mount immunostaining. Primary antibodies were selected based on sequence conservation of epitopes between skate and mammalian BK and Cav1.3 channels. Nuclei were counterstained with DAPI. Confocal z-stacks of 1-2 micron optical sections spanning up to 100 microns were acquired at 40x magnification through individual alveoli of the ampulla.

A high magnification optical section across the involuted wall of an alveolus is selected to illustrate notable features of BK channel $\alpha$ subunit expression and localization (Figure 1; Supplemental Movie 1). Anti-BK $\alpha$ rabbit polyclonal antibody PA1923 shows strong reactivity in disks (Figure 1A) that colocalize with DAPI nuclear staining of chromatin (Figure 1B, C). The nuclear expression of BK detected by PA1923 varies across the alveolar wall: the strongest BK expression in nuclear disks is present in cells one layer removed from the alveolar lumen (Figure 1C, gradient of pink). BK expression was also detected in hexagons, which we interpret as plasma membrane expression captured in cross-section (Figure 1A, C). We observed that cells with prominent hexagonal plasma membrane BK expression exhibited relatively lower levels of nuclear BK expression (Figure 1A). We independently detected BK expression using mouse monoclonal antibody MaxiK $\alpha$ (Figure 1D). MaxiK $\alpha$ also detected BK expression in disks (colocalizing with DAPI) and showed stronger immunoreactivity than PA1-923 in 
punctate foci and short spikes pointing into the alveolar lumen, which we interpret to represent BK channel expression in sensory kinocilia (Figure 1D-F, "k"). MaxiKa also showed stronger reactivity than PA1-923 at the basal, outer surfaces of alveoli where ribbon synapses of electroreceptor cells connect to the afferent nerves (Figure 1D-F, "rs").

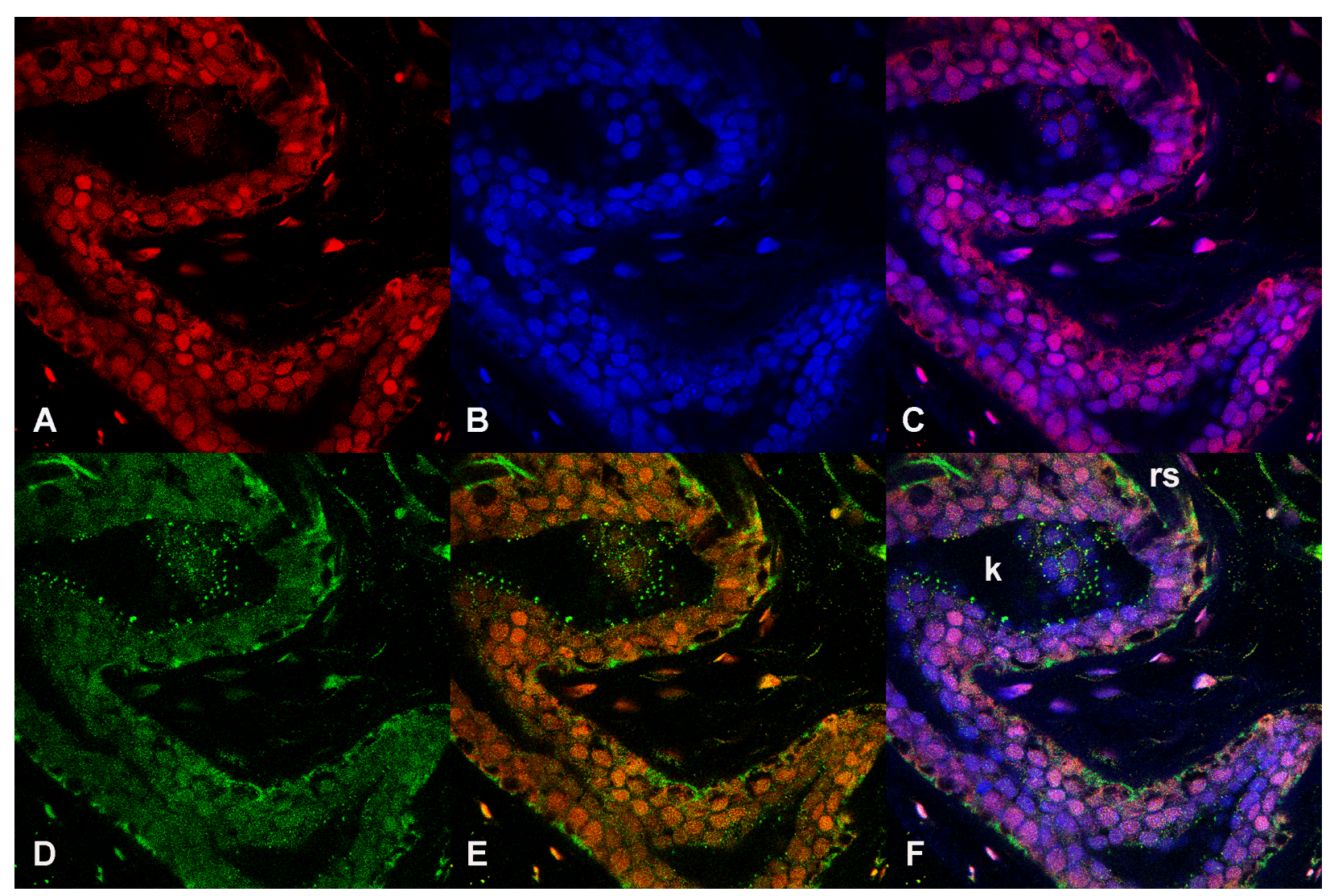

Figure 1: BK channels are present in nuclei and plasma membranes of skate ampullary electroreceptor. A) BK antibody PA1-923 (rabbit polyclonal). B) DAPI nuclear stain. C) Merge of A and B. D) BK antibody MaxiKa (mouse monoclonal). E) Merge of $A$ and $D . F)$ Merge of $A, B$ and $D$.

Colocalization of BK channels with nuclear lamins was next investigated. We combined rabbit anti-BK (PA1-923) with mouse anti-lamin A/C (Figure 2; Supplemental 
Movie 2). BK immunoreactivity was prominent in nuclear disks (Figure 2A,C,F). Lamin A/C immunoreactivity was present in disks (Figure 2D) that colocalized with DAPI nuclear staining (Figure 2B) and BK expression (Figure 2E,F). We detected lamin B1 using a rabbit antibody and combined this with anti-BK mouse MaxiK $\alpha$ (Figure 3; Supplemental Movie 3). BK immunoreactivity was prominent in rings (Figure 3A) surrounding nuclei (Figure 3B,C,F). Lamin B1 immunoreactivity marks inner nuclear membranes as rings (Figure 3D) and colocalizes precisely with BK immunoreactivity (Figure 3E,F) as showed by the pixel color shift of green + red to yellow. The arrow (Figure 3F) marks one of several puncta with strong BK and moderate lamin B1 immunoreactivity, which we interpret to represent the base of a kinocilia where it merges with the nuclear envelope (Supplemental Movie 3).

A

A

D
B

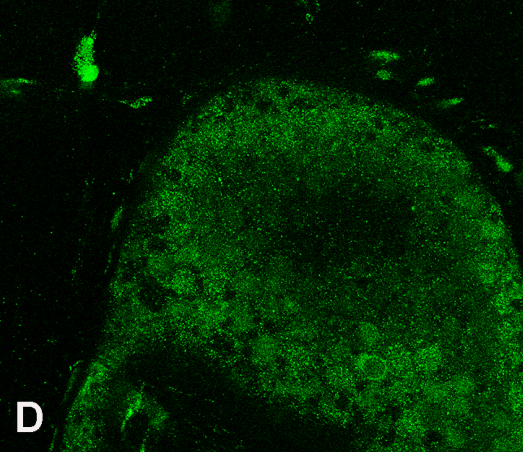

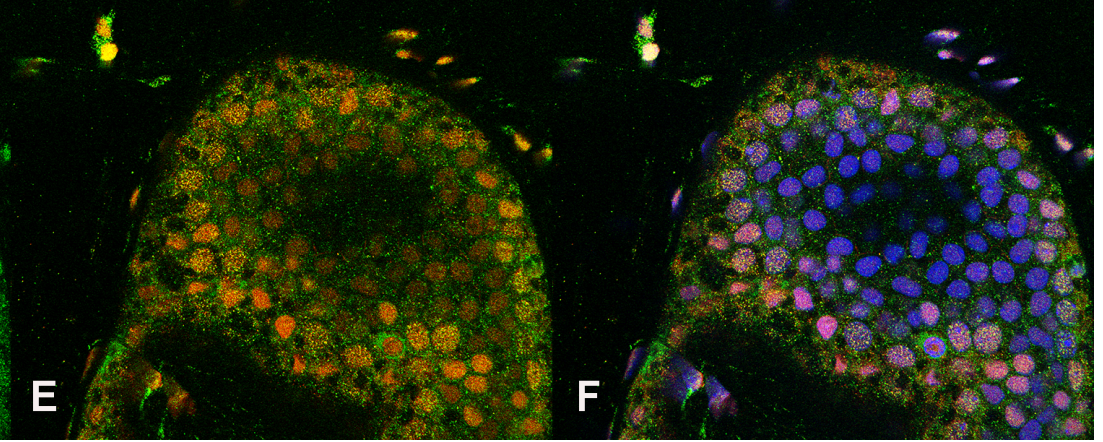

C 
Figure 2. BK channels colocalize with Lamin A/C. A) BK antibody (PA1-923, rabbit).

B) DAPI. C) Merge of A and B. D) Lamin A/C antibody (mouse monoclonal). E) Merge of $A$ and D. F) Merge of A, B and D.

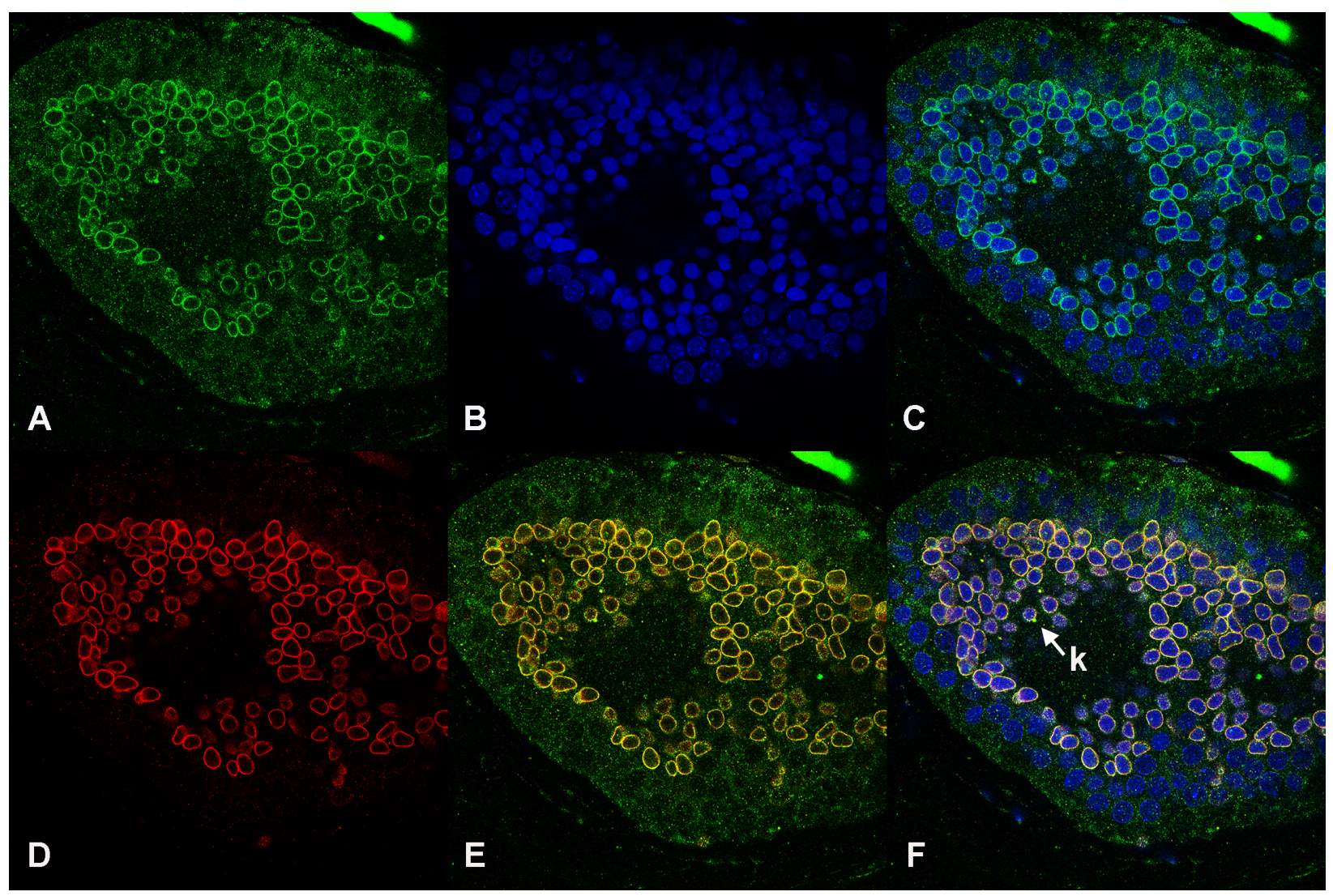

Figure 3. BK channels colocalize with Lamin B1. A) BK antibody (MaxiKa, mouse monoclonal). B) DAPI. C) Merge of A and B. D) Lamin B1 antibody (rabbit polyclonal). E) Merge of $A$ and D. F) Merge of A, B and D. "k" marks putative kinocilia base.

Expression of Cav1.3 was determined using mouse antibody LS-B4915 raised against rat Cav1.3 amino acids 859-875 (Figure 4; Supplemental Movies 5, 6). Skate Cav1.3 has 11/17 conserved amino acids in this epitope. Ampullae were coimmunostained with mouse anti- Cav1.3 (Figure 4A,G) and rabbit anti-BKa (PA1-923) 
(Figure 4D,J) and nuclei were stained with DAPI (Figure 4B,H). Cav1.3 is expressed in plasma membranes, ribbon synapses and kinocilia (Figure 4A,F, G,K; "rs", "k"). In contrast to BK channels (Figure 4D,J), there is little nuclear expression of Cav1.3. Colocalization of Cav1.3 and BK channels is seen in plasma membranes (Figure 4E,K). Cav1.3 expression is higher than BK expression at the outer, basal surfaces of electroreceptor cells where the ribbon synapses give rise to the afferent nerve fibers (Figure 4F,L; "rs"). Colocalization of Cav1.3 and BK channels is also concentrated at distinct punctae or short spikes which we interpret as kinocilia (Figure 4F,L, "k"). BK expression in disks overlying nuclei is seen in both the "wall" (Figure 4D-F) and "dome" (Figure 4J-L) views. In this specimen showing the alveolar "wall" (Figure 4A-F), there is again evidence of increasing BK nuclear expression in the layer of electroreceptor cells farthest from the lumen (Figure 4D-F). Additionally, the DAPI staining pattern differs between the two layers of nuclei in the wall of the alveolus (Figure 4B): those cells with greatest BK nuclear expression farthest from the lumen show mottled DAPI staining intercalated with BK staining (Figure 4F), whereas the nuclei closest to the lumen show uniform fine granular DAPI staining and little BK expression (except for single red dots). We propose the nuclear BK expression near chromatin may exist in nucleoplasmic envelopes connected to a nucleoplasmic reticulum [52], and contribute to regulation of gene expression, as previously reported [51]. Ultrastructural studies of Ampulla of Lorenzini by electron microscopy revealed that electroreceptor kinocilia intercalate between supporting cells that face the alveolar lumen [14]. 


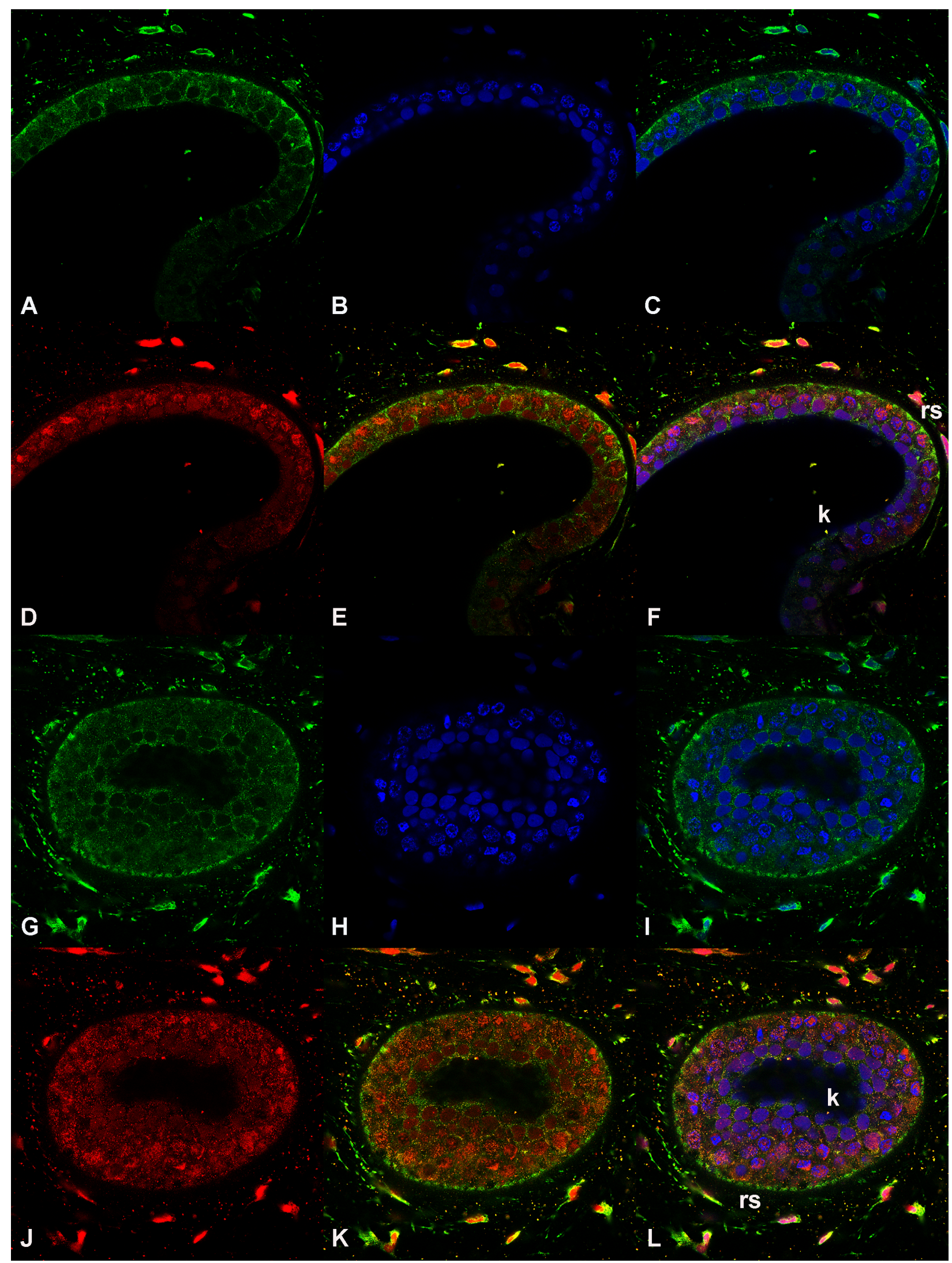


Figure 4: Expression of Cav1.3 and BK channels in skate ampullary electroreceptor. Wall A-F, Dome G-L. A,G) Cav1.3 antibody (mouse monoclonal LSB4915). B,H) DAPI. D,J) BK antibody (rabbit polyclonal PA1-923). C) Merge of A and B. E) Merge of $A$ and D. F) Merge of A, B and D. I) Merge of $G$ and H. K) Merge of $G$ and J. L) Merge of G, $H$ and J. "k" adjacent to luminal kinocilia, "rs" adjacent to basal ribbon synapses.

We analyzed skate BK channel sequences for potential nuclear localization signals using the program cNLS mapper [53]. The King (KJ756351) [27] and Bellono (KY355737) [29] sequences both contain a bipartite NLS, with different intervening linkers. These are KRIKKCGCKRLQDENPSVLSPKKKQRNG for King and KRIKKCGCKRPRYGYNGYLSTIQDENPSVLSPKKKQRNG for Bellono (note that Bellono has a 12 amino acid insert that is unique to their sequence: PRYGYNGYLSTI. These closely related, alternatively spliced bipartite NLS sequences are present at splice site 4 [38]. A 59-amino acid STREX exon alternatively spliced into this location [54] would substantially increase separation between the two clusters of basic $\mathrm{K}$ potentially attenuating functionality of the NLS. We analyzed BK channel proteomics and noted that the SVLSP sequence, or STLSP in human (NP_001309759.1), is frequently phosphorylated [55].

We used BlastP to search across species for proteins with sequence homology to the skate kcnma1 bipartite NLS (Supplemental Table). We found that the bipartite NLS with variable intervening linker in kcnma1 is highly conserved across diverse species. Vertebrates contain two clusters of basic amino acids, KRIKKCGCKR* 
upstream ( ${ }^{*}$ marks alternative splice 4, also known as c2) and KKKQRNG downstream. These sequences are absent in invertebrates, although Drosophila exhibits some homologous basic amino acids in these positions [34].

We evaluated this bipartite NLS for its ability to confer nuclear localization to skate BK channels transiently expressed in HEK293 cells. Skate kcnma1 cDNA cloned into pcDNA3.1 mammalian expression vector was a gift from Nicholas Bellono and David Julius [29]. Site directed mutations were generated within the bipartite NLS, by InFusion cloning. In the proximal portion of the bipartite NLS, two basic amino acids were mutated to alanine, $\mathrm{KR} \rightarrow \mathrm{AA}$. Separately, two serine residues within the linker domain that are sites of phosphorylation were mutated to alanine, SVLS $\rightarrow$ AVLA. HEK293 cells were either nontransfected, or transiently transfected with kcnma1 wild type (WT) expression vector, KR $\rightarrow$ AA or SVLS $\rightarrow$ AVLA mutations. After 40 h cells were fixed with paraformaldehyde, permeabilized with TX-100 and immunostained for BK channels (Slo1 antibody) and subcellular markers, Lamin B1 or Calnexin. Confocal microscopy was performed to characterize the subcellular localization of transfected, intracellular BK channels

Compared to control cells, HEK293 cells transfected with BK channels exhibited increased BK immunoreactivity in plasma membranes and intracellular compartments (Figure 5,6). Approximately 5-10\% of all transfected cells showed high levels of intracellular BK expression. HEK293 cells expressing WT BK channels showed substantial colocalization with DAPI and lamin B1, consistent with nuclear translocation (Figure 5F-J). In contrast, HEK293 cells transfected with KR $\rightarrow$ AA or SVLS->AVLA mutations showed high expression of BK channels adjacent to but not overlapping 
nuclei stained with DAPI and lamin B1 (Figure 5K-O and P-T). We note that accumulation of peri-nuclear-excluded BK channels caused indentations of the nuclei (arrowheads in Figure 5L,Q; Figure 6 L,Q).

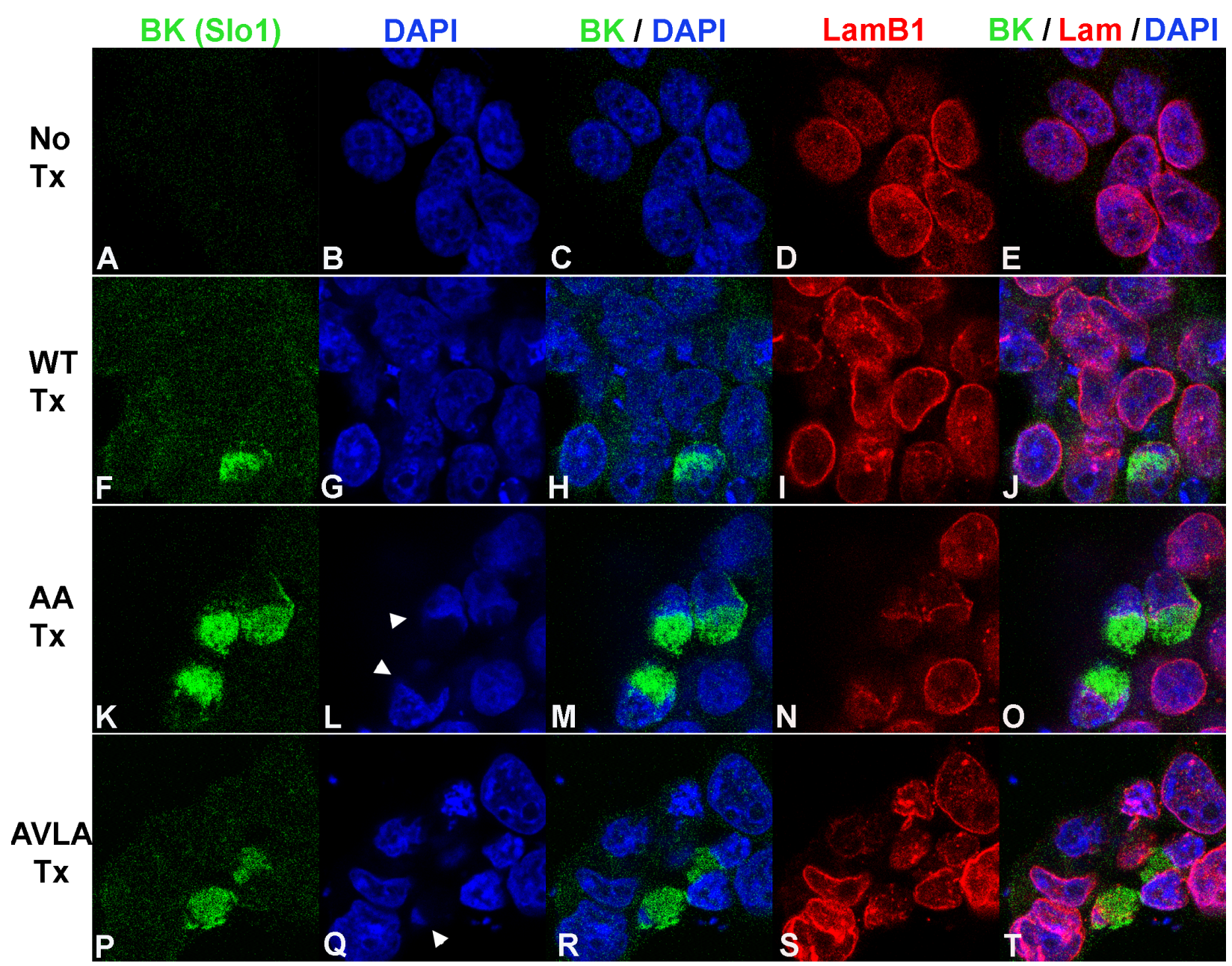

Figure 5. Localization of transfected skate BK channels and Lamin B1 in HEK293

cells. A-E: No transfection. A) anti-BK (Slo1 mouse monoclonal). B) DAPI. C) Merge of A and B. D) Lamin B1. E) Merge of A, B and D. F-J: WT transfection. F) anti-BK (Slo1). G) DAPI. H) Merge of F and G. I) Lamin B1 (rabbit polyclonal). J) Merge of F, G and I. K-O: AA mutation transfection. K) anti-BK (Slo1). L) DAPI. M) Merge of K and L. N) Lamin B1. O) Merge of K, L and N. P-T: AVLA mutation transfection. P) anti-BK (Slo1). 
Q) DAPI. R) Merge of $P$ and $Q$. S) Lamin B1. T) Merge of P, $Q$ and S.

High intracellular expression of BK channels adjacent to nuclei suggested localization in endoplasmic reticulum (ER). Calnexin is an integral membrane protein chaperone that localizes to and is used as a marker of the ER [56]. BK-transfected HEK cells were immunostained with anti-BK and anti-Calnexin antibodies (Figure 6). HEK293 cells expressing WT BK channels showed higher levels of colocalization with DAPI than with calnexin, consistent with nuclear translocation (Figure 6 F-J). In contrast, HEK293 cells transfected with $\mathrm{KR} \rightarrow \mathrm{AA}$ or SVLS->AVLA mutations showed substantial colocalization with calnexin, and not with DAPI, consistent with retention of BK channels in the ER (Figure 6, K-O and P-T). Again, we noted that accumulation of peri-nuclearexcluded BK channels caused indentations of the nuclei (Figure $6 \mathrm{Q}, \mathrm{S}$ ). 


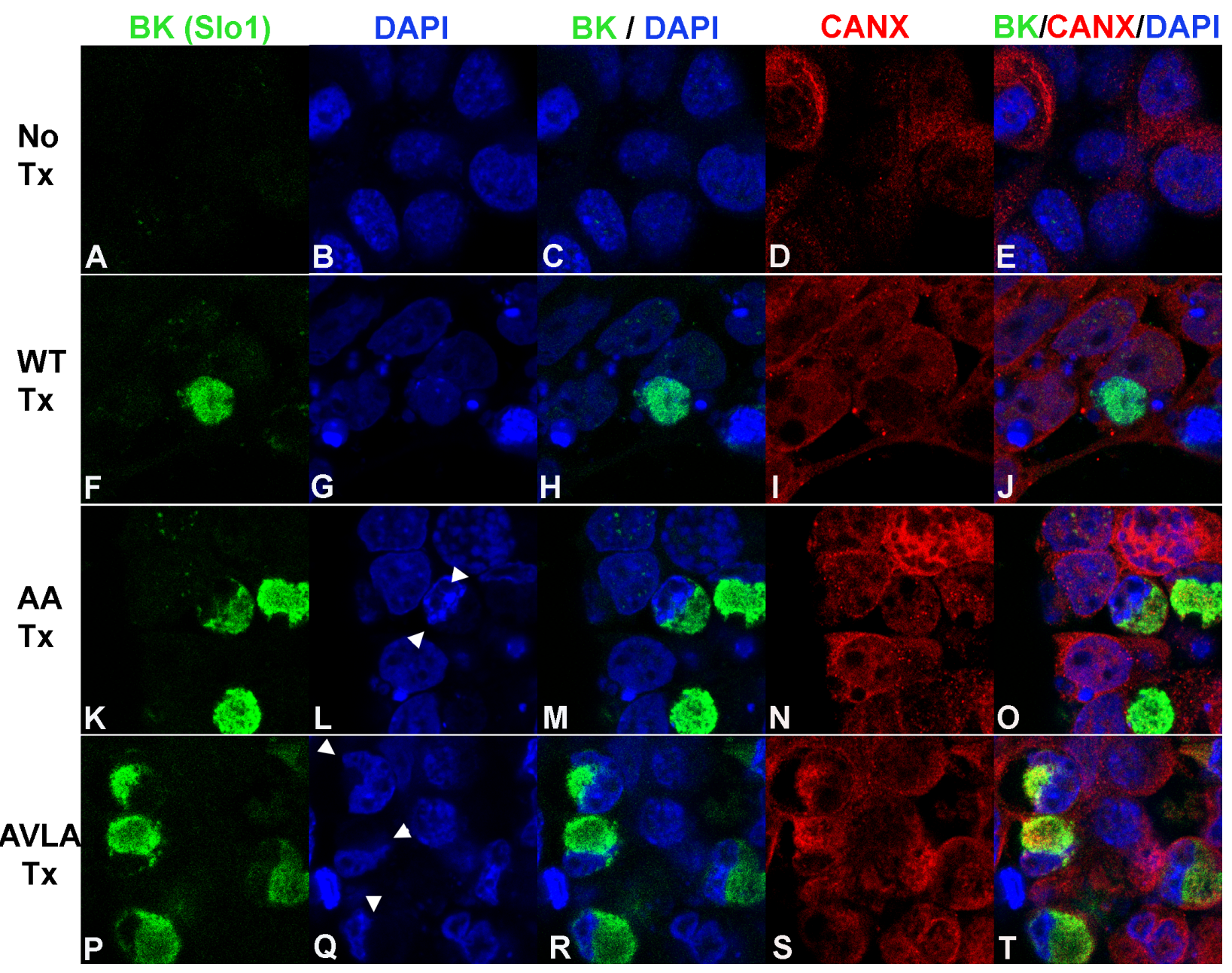

Figure 6. Localization of transfected skate BK channels and Calnexin in HEK293

cells. A-E: No transfection. A) anti-BKa (Slo1). B) DAPI. C) Merge of A and B. D)

Calnexin. E) Merge of A, B and D. F-J: WT transfection. F) anti-BK (Slo1, mouse). G)

DAPI. H) Merge of F and G. I) Calnexin (rabbit polyclonal). J) Merge of F, G and I. K-O:

AA mutation transfection. K) anti-BK (Slo1). L) DAPI. M) Merge of $\mathrm{K}$ and L. N) Calnexin.

O) Merge of K, L and N. P-T: AVLA mutation transfection. P) anti-BK (Slo1). Q) DAPI,

R) Merge of $P$ and $Q$. S) Calnexin. T) Merge of $P, Q$ and $S$. 
We counted HEK293 cells with high intracellular expression of BK channels and determined the subcellular localization pattern in relationship to WT, KR $\rightarrow$ AA or SVLS>AVLA mutations. The results are shown in Figure 7. WT BK channels translocate into the nucleus at $69 \%$ frequency, whereas mutations $K R \rightarrow A A$ or SVLS $\rightarrow$ AVLA significantly reduced nuclear translocation to $\sim 15 \%$. Compared to WT-transfected cells, SVLS $\rightarrow$ AVLA mutant transfected cells showed higher expression of intracellular BK channels $(\sim 10 \%)$, and greater survival after transient transfections.

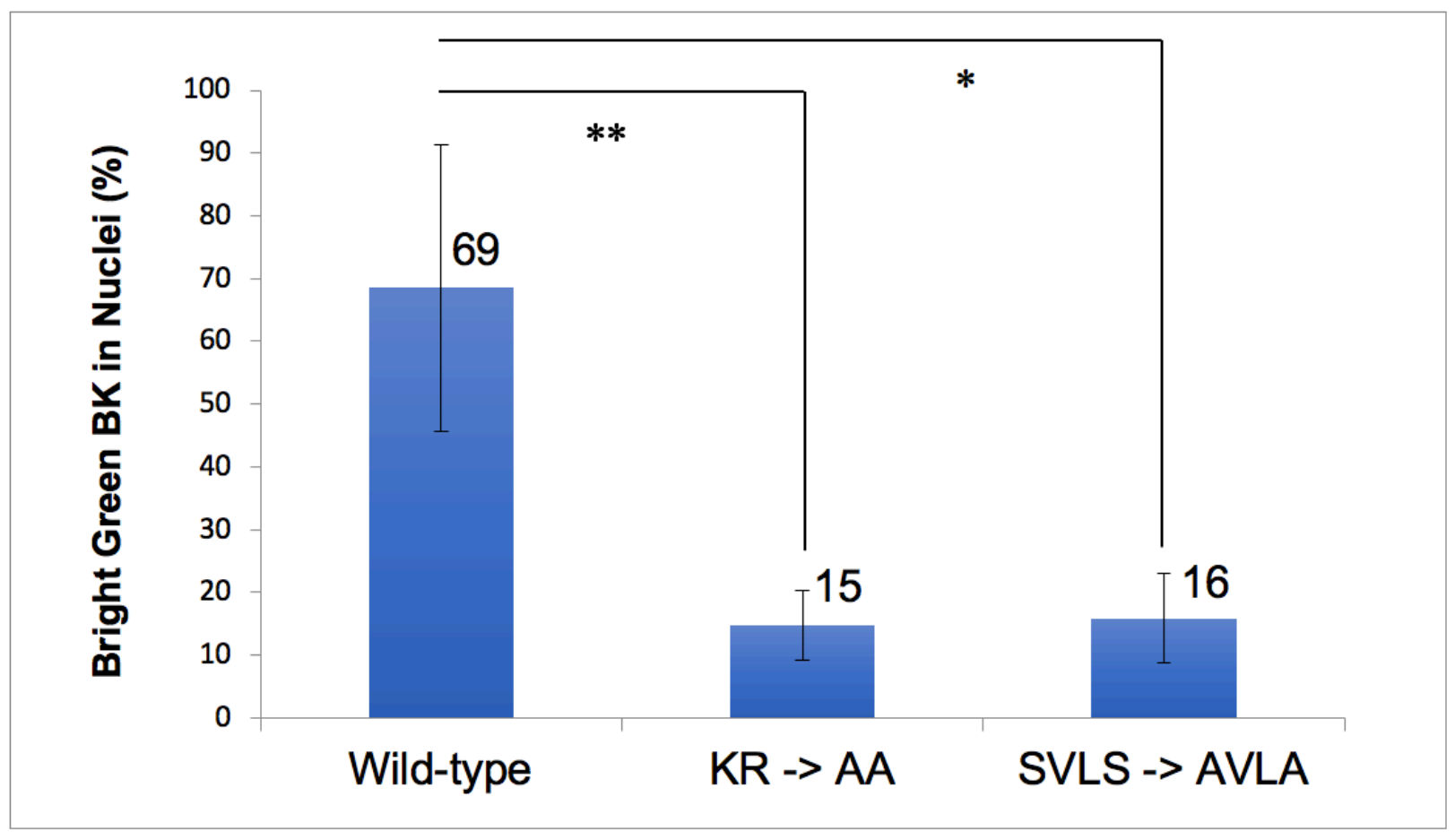

Figure 7. Localization of transfected skate BK channels in HEK293 cells.

Significance of the differences between Wild Type, AA or AVLA mutation transfections was determined using one-way ANOVA with Tukey correction. $p<0.05\left({ }^{*}\right),<0.01{ }^{* *}$ ).

We conclude that skate kcnma1 BKa channel contains a bipartite NLS, in which specific mutations attenuate nuclear translation. 


\section{Discussion}

Confocal microscopy of skate ampullae unexpectedly revealed strong expression of BK channels in nuclei of electroreceptor cells. Across an alveolus we detected different patterns of BK channel expression: cells with strong nuclear staining showed light plasma membrane expression and reciprocally, cells with prominent plasma membrane expression showed low levels of nuclear expression. We infer that BK channels may partition between plasma membranes and intracellular membranes such as nuclear envelopes, nucleoplasmic reticulum [52] or endoplasmic reticulum. In elegant studies, Kv10.1 channels were shown to be inserted into plasma membranes and available for surface biotinylation by extracellular BirA enzyme and subsequently trafficked to inner nuclear membranes where they were determined by single channel recording to be functional [33]. We suggest by analogy that electroreceptor BK channels located in intracellular membrane compartments and plasma membranes are likely to be electrically functional.

Examination of the skate kcnma1 sequences using cNLS mapper software identified clusters of basic amino acids likely to operate as a bipartite or two monopartite NLS. Notably the bipartite NLS occurs across splice site 4 [38], also identified as c2 [36]. Insertion here of alternative exons including the STREX exon, increases the separation between the two clusters of basic amino acids, and may disrupt the function as an NLS. A cross-species comparison of kcnma1 sequences demonstrates high conservation of the bipartite NLS in all vertebrates. Additionally, there is high species conservation of the phosphorylation substrates threonine and serine immediately before

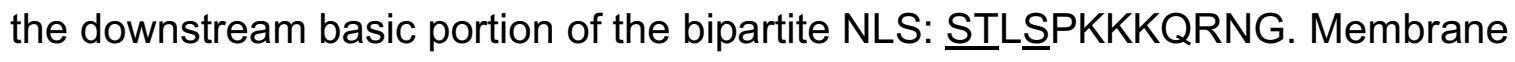


embedded proteins may be transported to the inner nuclear membrane through interactions of internal NLS with karophyrins (reviewed by [57]). The "SRKR" exon insertion at splice site 1 , modulated by circadian rhythm, affects plasma membrane BK channel activation [37]. This insertion adds three basic amino acids 84 residues upstream of the mouse kcnma1 bipartite NLS and creates an additional NLS that might affect nuclear trafficking; however, BK intracellular localization was not characterized.

Heterologous expression of kcnma1 BKa subunits in HEK293 cells is an established method for characterizing electrophysiologic properties of BK $\alpha$ splice variants and functional consequences of additional BK beta subunits. Here, we used heterologous expression of skate kcnma1 wild type [29] or mutated cDNAs in HEK293 cells to characterize intracellular BK $\alpha$ channel localization and regulation by the putative NLS. BK channel trafficking is undoubtedly complex and likely to depend on multiple addresses and post-translational modifications. For this study we chose not to fuse fluorescent proteins to BK $\alpha$ to minimize potential misfolding or mis-trafficking of the chimeric proteins. We performed BK localization analysis in kcnma1-transfected HEK293 cells after fixation, permeabilization and immunostaining similar to the skate ampulla. Our results establish that a fraction of transfected skate BK channels are expressed in the nucleus of HEK293 cells. Mutation of either, two basic amino acids in the first portion of the NLS or two serine residues upstream of the second portion of the NLS, independently attenuated nuclear localization of BK channels.

Numerous questions are raised by this study. What functional role may be served by BK channels in nuclei? Do nuclear BK channels contribute to calcium-regulated gene expression, if yes, which genes are most directly affected? Li et al [51] showed that BK 
channels in the nuclear envelope of hippocampal cells are functional and sensitive to pharmacologic inhibition by paxilline. Paxilline treatment of isolated nuclei caused depolarization of the nucleoplasm relative to the perinuclear lumen. Nuclear BK channels regulated the influx of calcium from the perinuclear space into the nucleoplasm. Paxilline-inhibition of nuclear BK channels produced transient increases in calcium in the nucleoplasm, and this nuclear calcium signaling affected calciumdependent gene transcription, neuronal activity and dendritic arborization.

What regulates the partitioning of BK channels between nuclear envelopes and plasma membranes? Hutchinson-Gilford progeria syndrome (HGPS) manifests as premature aging and is caused by genetic mutations in the nuclear envelope protein lamin A. Electrophysiologic studies of human dermal fibroblasts from HGPS patients exhibited higher plasma membrane expression and larger outward potassium currents than from healthy young subjects [58]. These results suggest that functional BK channels that normally partition between nuclear envelopes and plasma membranes are forced into plasma membranes when nuclear envelopes are genetically disrupted. Dysregulation of plasma membrane BK channel expression may affect cell electrical excitability, influencing signal pathways important in aging, growth and proliferation [59].

How does plasma membrane expression of BK channels affect cell excitability and electroreceptor oscillation frequency and tuning? Miranda-Rottman et al demonstrated positional gradients of BK alternative splicing in auditory hair cells along the chicken cochlea. Our study suggests that alternative splicing at site 4 within the bipartite NLS may regulate the balance between BK channel nuclear localization and 
plasma membrane density thereby influencing cell excitability and frequency responsiveness [42].

How dynamic is the trafficking, and recycling of specific BK channels between nuclei and plasma membranes? Kinocilia are specialized structures scaffolded by microtubules and enveloped by plasma membrane that contain extremely high densities of signaling receptors including ion channels $[60,61]$. The geometry of the cilium leads to a volume 5000-fold lower than the cytoplasm and enables signal amplification and quantal detection in sensory transduction. Cilia are anchored to basal bodies which operate as microtubule organizing centers, similar to centrioles [62]. Joukov et al [63] describe a physical connection between basal body apparatus and nucleus-associated microtubule organizing center as a means for communication between extracellular and intracellular domains. Our study suggests that BK channel partitioning between plasma membranes, including cilia, and nuclear envelopes may be regulated dynamically through posttranslational modifications within the bipartite NLS.

If BK trafficking is regulated by phosphorylation/dephosphorylation at the SVLS sequence in the bipartite NLS, what enzymes may be involved in these posttranslational modifications? Phosphorylation of Ser385 in the EBNA-1 NLS promoted nuclear import by increasing its affinity for karophyrin importin $\alpha 5$ [64]. What activates these enzymes? Are these enzymes potentially novel drug targets that might modulate BK trafficking and thereby cell signaling? 


\section{Materials and Methods}

\section{Dissection and whole mount immunostaining}

Adult skates (Leucoraja erinacea) were purchased from Marine Biological Labs in Woods Hole, MA and shipped alive in chilled seawater. Animals were killed instantly by pithing of the brain and spinal cord. The ampullae were dissected with scissors from behind the gill slits. Approximately 200 ampullae with a short section of ampullary canal and afferent nerve were dissected in PBS under a stereomicroscope and cleaned of connective tissue. Ampulla were fixed in 1\% paraformaldehyde in PBS for $1 \mathrm{~h}$, then rinsed two times in PBS and stored for up to 1 week.

Whole mount staining was performed in $0.5 \mathrm{ml}$ microfuge tubes in 100 microliters of solution, then blocked was with 10\% FBS and 1\% Triton X-100 detergent. Staining was performed with mouse and rabbit primary antibodies at final concentrations of 4 to $20 \mu \mathrm{g} / \mathrm{ml}$ in $0.1 \% \mathrm{FBS}$ and $0.1 \% \mathrm{TX}-100$ for $48 \mathrm{~h}$, followed by 3 serial washes with PBS $-0.1 \%$ TX-100. Secondary antibodies were applied at 1:100 dilution; anti-mouse $\lg G(H+L)$ or $\lg G 2 \alpha$ Alexa 488 or anti-rabbit Alexa 594. Nuclei were counterstained with DAPI.

Primary antibodies used were anti-BKa: KCNMA1 PA1-923 Invitrogen /ThermoFisher, rabbit polyclonal IgG, raised against synthetic peptide of human KCNMA1: $T_{945}$ ELVNDTNVQFLDQDDD $_{961}$, skate epitope is identical at $17 / 18$ positions, underlined: TELVNDSNVQFLDQDDD. MaxiKa (B-1) sc-374142 Santa Cruz Biotechnology, mouse monoclonal lgG2b immunogen amino acids 937-1236 at Cterminus of human MaxiKa. Anti-Slo1, clone L6/60 MABN70 Millipore, mouse monoclonal IgG2a, immunogen is recombinant protein corresponding to segment S9- 
S10 of mouse Slo1 (NP_034740). For Cav1.3 cacna1d: LS-B4915 LifeSpan Biosciences, mouse monoclonal lgG2a, immunogen is fusion protein encoding amino acids 8590875 of rat Cav1.3 (Accession P27732): DNK------VTIDDYQEEAEDKD, skate epitope is conserved at 11 of 16 positions, underlined: $\underline{D} \underline{R}$ ILTGTQ $\underline{V}$ IDD-Q--DEDKD. Lamin A/C: NBP2-59937 Novus Biologicals, mouse monoclonal IgG3. Lamin B1 Ab16048 AbCam, immunogen synthetic peptide corresponding to mouse Lamin B1 amino acids 400-500 conjugated to keyhole limpet hemocyanin. Calnexin HPA009433 Sigma Atlas Prestige Antibodies, rabbit polyclonal lgG,

\section{https://www.proteinatlas.org/ENSG00000127022-CANX/antibody.}

Confocal imaging was performed using Zeiss LSM 880. Objective lenses used were apochromatic 10x/NA 0.45 and 40x/NA 1.2 glycerol immersion infinity/0.15-0.19. Z-stacks of up to 100 microns were collected, using separate tracks to avoid cross contamination of colors. Image files were processed using Fiji (Image J, NIH). Site-directed mutagenesis

Skate BK channel in mammalian expression vector pcDNA 3.1 - kcnma1 was a generous gift from Bellono and Julius [29](Accession KY355737.1). Site directed mutations were generated using InFusion cloning system (ThermoFisher) with designated primers (nucleotide changes from WT in capital letters):

KCNMA1_NLSmuKR655AA_1954plus 5'tgtgggtgtGCAGCAccaagatatggctataatggatatctcagcac, and KCNMA1_NLSmuKR655AA_1962minus 5'GGTGCTGCacacccacatttcttaattctctttgtatctgtg; KCNMA1_NLS_SVLS674AVLA_2017plus 5'- 
ccaGcagtgctgGctcccaaaaaaaagcaacggaacgggggc, and

KCNMA1_NLS_SVLS674AVLA_2033minus 5'-

ggagCcagcactgCtgggttttcatcttgaattgtgctgag.

Mutagenesis was confirmed by DNA sequencing. WT; KR $\rightarrow$ AA mutation and

SVLS $\rightarrow$ AVLA mutations.

\section{Immunofluorescence staining}

HEK293 cells were transfected on multiwell imaging slides. Between 300-700 ng of DNA plasmid was mixed with JetPrime lipid transfection agent and applied to cells at $50 \%$ confluency for $\sim 40 \mathrm{~h}$. Cells were fixed with 1\% PFA, and immunostained at 10 $\mu \mathrm{g} / \mathrm{ml}$ using anti-BK mouse monoclonal anti-Slo1 and rabbit polyclonal anti-Lamin B1 (nuclear envelope marker) or anti-Calnexin (ER marker [56]) antibodies. Nontransfected cells did not demonstrate prominent Slo1 staining. Transfected cells with prominent (intracellular) Slo1 staining were counted, and the fraction of those cells that exhibited overlap with DAPI nuclear stain was counted manually. The effect of sitedirected mutations on nuclear localization of skate BK channels was determined quantitatively.

\section{Acknowledgements}

We acknowledge the contributions of Alexis Froistad acquiring the initial confocal images of the skate ampulla. 


\section{References}

1. Meech RW. Calcium-dependent potassium activation in nervous tissues. Annu Rev Biophys Bioeng. 1978;7:1-18. Epub 1978/01/01. doi:

10.1146/annurev.bb.07.060178.000245. PubMed PMID: 352237.

2. N'Gouemo P. Targeting BK (big potassium) channels in epilepsy. Expert Opin Ther Targets. 2011;15(11):1283-95. Epub 2011/09/20. doi:

10.1517/14728222.2011.620607. PubMed PMID: 21923633; PubMed Central PMCID: PMCPMC3219529.

3. Treistman SN, Martin GE. BK Channels: mediators and models for alcohol tolerance. Trends Neurosci. 2009;32(12):629-37. Epub 2009/09/29. doi:

10.1016/j.tins.2009.08.001. PubMed PMID: 19781792; PubMed Central PMCID: PMCPMC4115799.

4. Qi XY, Diness JG, Brundel BJ, Zhou XB, Naud P, Wu CT, et al. Role of smallconductance calcium-activated potassium channels in atrial electrophysiology and fibrillation in the dog. Circulation. 2014;129(4):430-40. Epub 2013/11/06. doi: 10.1161/CIRCULATIONAHA.113.003019. PubMed PMID: 24190961.

5. Chang PC, Hsieh YC, Hsueh CH, Weiss JN, Lin SF, Chen PS. Apamin induces early afterdepolarizations and torsades de pointes ventricular arrhythmia from failing rabbit ventricles exhibiting secondary rises in intracellular calcium. Heart Rhythm. 2013;10(10):1516-24. Epub 2013/07/10. doi: 10.1016/j.hrthm.2013.07.003. PubMed PMID: 23835258; PubMed Central PMCID: PMCPMC3832504.

6. Hu X, Laragione T, Sun L, Koshy S, Jones KR, Ismailov, II, et al. KCa1.1 potassium channels regulate key proinflammatory and invasive properties of fibroblastlike synoviocytes in rheumatoid arthritis. The Journal of biological chemistry. 2012;287(6):4014-22. Epub 2011/11/15. doi: 10.1074/jbc.M111.312264. PubMed PMID: 22074915; PubMed Central PMCID: PMCPMC3281680.

7. Tanner MR, Hu X, Huq R, Tajhya RB, Sun L, Khan FS, et al. KCa1.1 inhibition attenuates fibroblast-like synoviocyte invasiveness and ameliorates disease in rat models of rheumatoid arthritis. Arthritis Rheumatol. 2015;67(1):96-106. Epub 2014/09/25. doi: 10.1002/art.38883. PubMed PMID: 25252152; PubMed Central PMCID: PMCPMC4280298.

8. Tanner MR, Pennington MW, Laragione T, Gulko PS, Beeton C. KCa1.1 channels regulate beta1-integrin function and cell adhesion in rheumatoid arthritis fibroblast-like synoviocytes. FASEB J. 2017;31(8):3309-20. Epub 2017/04/22. doi: 10.1096/fj.201601097R. PubMed PMID: 28428266; PubMed Central PMCID: PMCPMC5503717.

9. Tajhya RB, Hu X, Tanner MR, Huq R, Kongchan N, Neilson JR, et al. Functional $\mathrm{KCa} 1.1$ channels are crucial for regulating the proliferation, migration and differentiation of human primary skeletal myoblasts. Cell Death Dis. 2016;7(10):e2426. Epub 2016/10/21. doi: 10.1038/cddis.2016.324. PubMed PMID: 27763639; PubMed Central PMCID: PMCPMC5133989.

10. Baker CVH, Modrell MS. Insights into Electroreceptor Development and Evolution from Molecular Comparisons with Hair Cells. Integr Comp Biol. 2018;58(2):329-40. Epub 2018/05/31. doi: 10.1093/icb/icy037. PubMed PMID: 29846597; PubMed Central PMCID: PMCPMC6927855. 
11. Gauthier ARG, Whitehead DL, Tibbetts IR, Cribb BW, Bennett MB. Morphological comparison of the ampullae of Lorenzini of three sympatric benthic rays. J Fish Biol. 2018;92(2):504-14. Epub 2018/02/13. doi: 10.1111/jfb.13531. PubMed PMID: 29431219.

12. Josberger EE, Hassanzadeh P, Deng Y, Sohn J, Rego MJ, Amemiya CT, et al. Proton conductivity in ampullae of Lorenzini jelly. Sci Adv. 2016;2(5):e1600112. Epub 2016/07/08. doi: 10.1126/sciadv.1600112. PubMed PMID: 27386543; PubMed Central PMCID: PMCPMC4928922.

13. Sejnowski TJ, Yodlowski ML. A freeze-fracture study of the skate electroreceptor. J Neurocytol. 1982;11(6):897-912. Epub 1982/12/01. doi: 10.1007/bf01148307. PubMed PMID: 7153788.

14. Wueringer BE, Tibbetts IR, Whitehead DL. Ultrastructure of the ampullae of Lorenzini of Aptychotrema rostrata (Rhinobatidae). Zoomorphology. 2009;128:45-52. doi: https://doi.org/10.1007/s00435-008-0073-5.

15. Waltman B. Electrical properties and fine structure of the ampullary canals of Lorenzini. Acta Physiol Scand Suppl. 1966;264:1-60. Epub 1966/01/01. PubMed PMID: 5911901.

16. Kalmijn AJ. The electric sense of sharks and rays. J Exp Biol. 1971;55(2):371-83. Epub 1971/10/01. PubMed PMID: 5114029.

17. Obara S, Bennett MV. Mode of operation of ampullae of Lorenzini of the skate, Raja. J Gen Physiol. 1972;60(5):534-57. Epub 1972/11/01. doi: 10.1085/jgp.60.5.534. PubMed PMID: 4345438; PubMed Central PMCID: PMCPMC2226088.

18. Lu J, Fishman HM. Interaction of apical and basal membrane ion channels underlies electroreception in ampullary epithelia of skates. Biophys J. 1994;67(4):152533. Epub 1994/10/01. doi: 10.1016/S0006-3495(94)80626-5. PubMed PMID: 7529586; PubMed Central PMCID: PMCPMC1225515.

19. Lu J, Fishman HM. Localization and function of the electrical oscillation in electroreceptive ampullary epithelium from skates. Biophys J. 1995;69(6):2458-66. Epub 1995/12/01. doi: 10.1016/S0006-3495(95)80116-5. PubMed PMID: 8599652; PubMed Central PMCID: PMCPMC1236483.

20. Lu J, Fishman HM. Ion channels and transporters in the electroreceptive ampullary epithelium from skates. Biophys J. 1995;69(6):2467-75. Epub 1995/12/01. doi: 10.1016/S0006-3495(95)80117-7. PubMed PMID: 8599653; PubMed Central PMCID: PMCPMC1236484.

21. Clusin W, Spray DC, Bennett MV. Activation of a voltage-insensitive conductance by inward calcium current. Nature. 1975;256(5516):425-7. Epub 1975/07/31. doi: 10.1038/256425a0. PubMed PMID: 1143347.

22. Clusin WT, Bennett MV. Calcium-activated conductance in skate electroreceptors: current clamp experiments. J Gen Physiol. 1977;69(2):121-43. Epub 1977/02/01. doi: 10.1085/jgp.69.2.121. PubMed PMID: 190338; PubMed Central PMCID: PMCPMC2215012.

23. Clusin WT, Bennett MV. Calcium-activated conductance in skate electroreceptors: voltage clamp experiments. J Gen Physiol. 1977;69(2):145-82. Epub 1977/02/01. doi: 10.1085/jgp.69.2.145. PubMed PMID: 402438; PubMed Central PMCID: PMCPMC2215014. 
24. Clusin WT, Bennett MV. The ionic basis of oscillatory responses of skate electroreceptors. J Gen Physiol. 1979;73(6):703-23. Epub 1979/06/01. doi: 10.1085/jgp.73.6.703. PubMed PMID: 479811; PubMed Central PMCID: PMCPMC2215204.

25. Clusin WT, Bennett MV. The oscillatory responses of skate electroreceptors to small voltage stimuli. J Gen Physiol. 1979;73(6):685-702. Epub 1979/06/01. doi: 10.1085/jgp.73.6.685. PubMed PMID: 479810; PubMed Central PMCID: PMCPMC2215210.

26. Modrell MS, Lyne M, Carr AR, Zakon HH, Buckley D, Campbell AS, et al. Insights into electrosensory organ development, physiology and evolution from a lateral line-enriched transcriptome. Elife. 2017;6. Epub 2017/03/28. doi: 10.7554/eLife.24197. PubMed PMID: 28346141; PubMed Central PMCID: PMCPMC5429088.

27. King BL, Shi LF, Kao P, Clusin WT. Calcium activated $\mathrm{K}(+)$ channels in the electroreceptor of the skate confirmed by cloning. Details of subunits and splicing. Gene. 2016;578(1):63-73. Epub 2015/12/22. doi: 10.1016/j.gene.2015.12.010. PubMed PMID: 26687710; PubMed Central PMCID: PMCPMC4724458.

28. Clusin WT, Wu TH, Shi LF, Kao PN. Further studies of ion channels in the electroreceptor of the skate through deep sequencing, cloning and cross species comparisons. Gene. 2019;718:143989. Epub 2019/07/22. doi:

10.1016/j.gene.2019.143989. PubMed PMID: 31326551.

29. Bellono NW, Leitch DB, Julius D. Molecular basis of ancestral vertebrate electroreception. Nature. 2017;543(7645):391-6. Epub 2017/03/07. doi: 10.1038/nature21401. PubMed PMID: 28264196; PubMed Central PMCID: PMCPMC5354974.

30. Checchetto V, Teardo E, Carraretto L, Leanza L, Szabo I. Physiology of intracellular potassium channels: A unifying role as mediators of counterion fluxes? Biochim Biophys Acta. 2016;1857(8):1258-66. Epub 2016/03/13. doi: 10.1016/j.bbabio.2016.03.011. PubMed PMID: 26970213.

31. Capera J, Serrano-Novillo C, Navarro-Perez M, Cassinelli S, Felipe A. The Potassium Channel Odyssey: Mechanisms of Traffic and Membrane Arrangement. Int J Mol Sci. 2019;20(3). Epub 2019/02/13. doi: 10.3390/ijms20030734. PubMed PMID: 30744118; PubMed Central PMCID: PMCPMC6386995.

32. Ouadid-Ahidouch $\mathrm{H}$, Ahidouch A, Pardo LA. Kv10.1 K(+) channel: from physiology to cancer. Pflugers Arch. 2016;468(5):751-62. Epub 2016/01/09. doi: 10.1007/s00424-015-1784-3. PubMed PMID: 26743871.

33. Chen Y, Sanchez A, Rubio ME, Kohl T, Pardo LA, Stuhmer W. Functional $\mathrm{K}(\mathrm{v}) 10.1$ channels localize to the inner nuclear membrane. PloS one. 2011;6(5):e19257. Epub 2011/05/12. doi: 10.1371/journal.pone.0019257. PubMed PMID: 21559285; PubMed Central PMCID: PMCPMC3086910.

34. Tseng-Crank J, Foster CD, Krause JD, Mertz R, Godinot N, DiChiara TJ, et al. Cloning, expression, and distribution of functionally distinct $\mathrm{Ca}(2+)$-activated $\mathrm{K}+$ channel isoforms from human brain. Neuron. 1994;13(6):1315-30. Epub 1994/12/01. doi: 10.1016/0896-6273(94)90418-9. PubMed PMID: 7993625.

35. Pyott SJ, Glowatzki E, Trimmer JS, Aldrich RW. Extrasynaptic localization of inactivating calcium-activated potassium channels in mouse inner hair cells. J Neurosci. 
2004;24(43):9469-74. Epub 2004/10/29. doi: 10.1523/JNEUROSCI.3162-04.2004. PubMed PMID: 15509733; PubMed Central PMCID: PMCPMC6730162.

36. Chen L, Tian L, MacDonald SH, McClafferty H, Hammond MS, Huibant JM, et al. Functionally diverse complement of large conductance calcium- and voltage-activated potassium channel (BK) alpha-subunits generated from a single site of splicing. The Journal of biological chemistry. 2005;280(39):33599-609. Epub 2005/08/06. doi: 10.1074/jbc.M505383200. PubMed PMID: 16081418.

37. Shelley C, Whitt JP, Montgomery JR, Meredith AL. Phosphorylation of a constitutive serine inhibits BK channel variants containing the alternate exon "SRKR". J Gen Physiol. 2013;142(6):585-98. Epub 2013/11/28. doi: 10.1085/jgp.201311072. PubMed PMID: 24277602; PubMed Central PMCID: PMCPMC3840924.

38. Miranda-Rottmann S, Kozlov AS, Hudspeth AJ. Highly specific alternative splicing of transcripts encoding BK channels in the chicken's cochlea is a minor determinant of the tonotopic gradient. Molecular and cellular biology. 2010;30(14):364660. Epub 2010/05/19. doi: 10.1128/MCB.00073-10. PubMed PMID: 20479127; PubMed Central PMCID: PMCPMC2897565.

39. Kathiresan T, Harvey M, Orchard S, Sakai Y, Sokolowski B. A protein interaction network for the large conductance $\mathrm{Ca}(2+)$-activated $\mathrm{K}(+)$ channel in the mouse cochlea. Mol Cell Proteomics. 2009;8(8):1972-87. Epub 2009/05/09. doi: 10.1074/mcp.M800495MCP200. PubMed PMID: 19423573; PubMed Central PMCID: PMCPMC2722780.

40. Sokolowski B, Orchard S, Harvey M, Sridhar S, Sakai Y. Conserved BK channelprotein interactions reveal signals relevant to cell death and survival. PloS one. 2011;6(12):e28532. Epub 2011/12/17. doi: 10.1371/journal.pone.0028532. PubMed PMID: 22174833; PubMed Central PMCID: PMCPMC3235137.

41. Kyle BD, Braun AP. The regulation of BK channel activity by pre- and posttranslational modifications. Front Physiol. 2014;5:316. Epub 2014/09/10. doi: 10.3389/fphys.2014.00316. PubMed PMID: 25202279; PubMed Central PMCID: PMCPMC4141542.

42. Pyott SJ, Duncan RK. BK Channels in the Vertebrate Inner Ear. Int Rev Neurobiol. 2016;128:369-99. Epub 2016/05/31. doi: 10.1016/bs.irn.2016.03.016. PubMed PMID: 27238269.

43. Latorre R, Castillo K, Carrasquel-Ursulaez W, Sepulveda RV, Gonzalez-Nilo F, Gonzalez C, et al. Molecular Determinants of BK Channel Functional Diversity and Functioning. Physiological reviews. 2017;97(1):39-87. Epub 2016/11/04. doi: 10.1152/physrev.00001.2016. PubMed PMID: 27807200.

44. Lingle CJ, Martinez-Espinosa PL, Yang-Hood A, Boero LE, Payne S, Persic D, et al. LRRC52 regulates BK channel function and localization in mouse cochlear inner hair cells. Proceedings of the National Academy of Sciences of the United States of America. 2019;116(37):18397-403. Epub 2019/08/28. doi: 10.1073/pnas.1907065116. PubMed PMID: 31451634 ; PubMed Central PMCID: PMCPMC6744894.

45. Singh H, Lu R, Bopassa JC, Meredith AL, Stefani E, Toro L. MitoBK(Ca) is encoded by the Kcnma1 gene, and a splicing sequence defines its mitochondrial location. Proceedings of the National Academy of Sciences of the United States of America. 2013;110(26):10836-41. Epub 2013/06/12. doi: 10.1073/pnas.1302028110. PubMed PMID: 23754429; PubMed Central PMCID: PMCPMC3696804. 
46. Giraldez T, Hughes TE, Sigworth FJ. Generation of functional fluorescent BK channels by random insertion of GFP variants. J Gen Physiol. 2005;126(5):429-38. Epub 2005/11/02. doi: 10.1085/jgp.200509368. PubMed PMID: 16260837; PubMed Central PMCID: PMCPMC2266602.

47. Mazzanti M, DeFelice LJ, Cohn J, Malter H. Ion channels in the nuclear envelope. Nature. 1990;343(6260):764-7. Epub 1990/02/22. doi: 10.1038/343764a0. PubMed PMID: 2304551.

48. Fedorenko O, Yarotskyy V, Duzhyy D, Marchenko S. The large-conductance ion channels in the nuclear envelope of central neurons. Pflugers Arch. 2010;460(6):104550. Epub 2010/10/05. doi: 10.1007/s00424-010-0882-5. PubMed PMID: 20886229. 49. Singh $\mathrm{H}$, Stefani E, Toro L. Intracellular BK(Ca) (iBK(Ca)) channels. J Physiol. 2012;590(23):5937-47. Epub 2012/08/30. doi: 10.1113/jphysiol.2011.215533. PubMed PMID: 22930268; PubMed Central PMCID: PMCPMC3530108.

50. Li B, Gao TM. Functional Role of Mitochondrial and Nuclear BK Channels. Int Rev Neurobiol. 2016;128:163-91. Epub 2016/05/31. doi: 10.1016/bs.irn.2016.03.018. PubMed PMID: 27238264.

51. Li B, Jie W, Huang L, Wei P, Li S, Luo Z, et al. Nuclear BK channels regulate gene expression via the control of nuclear calcium signaling. Nat Neurosci. 2014;17(8):1055-63. Epub 2014/06/24. doi: 10.1038/nn.3744. PubMed PMID: 24952642; PubMed Central PMCID: PMCPMC4115017.

52. Malhas A, Goulbourne C, Vaux DJ. The nucleoplasmic reticulum: form and function. Trends Cell Biol. 2011;21(6):362-73. Epub 2011/04/26. doi: 10.1016/j.tcb.2011.03.008. PubMed PMID: 21514163.

53. Kosugi S, Hasebe M, Tomita M, Yanagawa H. Systematic identification of cell cycle-dependent yeast nucleocytoplasmic shuttling proteins by prediction of composite motifs. Proceedings of the National Academy of Sciences of the United States of America. 2009;106(25):10171-6. Epub 2009/06/13. doi: 10.1073/pnas.0900604106. PubMed PMID: 19520826 ; PubMed Central PMCID: PMCPMC2695404.

54. Shipston MJ, Duncan RR, Clark AG, Antoni FA, Tian L. Molecular components of large conductance calcium-activated potassium (BK) channels in mouse pituitary corticotropes. Mol Endocrinol. 1999;13(10):1728-37. Epub 1999/10/12. doi: 10.1210/mend.13.10.0355. PubMed PMID: 10517674.

55. Yan J, Olsen JV, Park KS, Li W, BildI W, Schulte U, et al. Profiling the phosphostatus of the BKCa channel alpha subunit in rat brain reveals unexpected patterns and complexity. Mol Cell Proteomics. 2008;7(11):2188-98. Epub 2008/06/25. doi:

10.1074/mcp.M800063-MCP200. PubMed PMID: 18573811; PubMed Central PMCID: PMCPMC2577206.

56. Williams DB. Beyond lectins: the calnexin/calreticulin chaperone system of the endoplasmic reticulum. J Cell Sci. 2006;119(Pt 4):615-23. Epub 2006/02/10. doi: 10.1242/jcs.02856. PubMed PMID: 16467570.

57. Katta SS, Smoyer CJ, Jaspersen SL. Destination: inner nuclear membrane. Trends Cell Biol. 2014;24(4):221-9. Epub 2013/11/26. doi: 10.1016/j.tcb.2013.10.006. PubMed PMID: 24268652.

58. Zironi I, Gavoci E, Lattanzi G, Virelli A, Amorini F, Remondini D, et al. BK channel overexpression on plasma membrane of fibroblasts from Hutchinson-Gilford progeria syndrome. Aging. 2018;10(11):3148-60. Epub 2018/11/07. doi: 
10.18632/aging.101621. PubMed PMID: 30398975; PubMed Central PMCID: PMCPMC6286842.

59. Toro L, Li M, Zhang Z, Singh H, Wu Y, Stefani E. MaxiK channel and cell signalling. Pflugers Arch. 2014;466(5):875-86. Epub 2013/10/01. doi: 10.1007/s00424013-1359-0. PubMed PMID: 24077696; PubMed Central PMCID: PMCPMC3969412. 60. Nachury MV. How do cilia organize signalling cascades? Philos Trans R Soc Lond B Biol Sci. 2014;369(1650). Epub 2014/07/23. doi: 10.1098/rstb.2013.0465. PubMed PMID: 25047619; PubMed Central PMCID: PMCPMC4113109.

61. Falk N, LosI M, Schroder N, Giessl A. Specialized Cilia in Mammalian Sensory Systems. Cells. 2015;4(3):500-19. Epub 2015/09/18. doi: 10.3390/cells4030500. PubMed PMID: 26378583; PubMed Central PMCID: PMCPMC4588048. 62. Moser JJ, Fritzler MJ, Ou Y, Rattner JB. The PCM-basal body/primary cilium coalition. Semin Cell Dev Biol. 2010;21(2):148-55. Epub 2009/07/14. doi: 10.1016/j.semcdb.2009.06.006. PubMed PMID: 19591955.

63. Joukov V, De Nicolo A. The Centrosome and the Primary Cilium: The Yin and Yang of a Hybrid Organelle. Cells. 2019;8(7). Epub 2019/07/13. doi: 10.3390/cells8070701. PubMed PMID: 31295970; PubMed Central PMCID: PMCPMC6678760.

64. Nardozzi JD, Lott K, Cingolani G. Phosphorylation meets nuclear import: a review. Cell Commun Signal. 2010;8:32. Epub 2010/12/25. doi: 10.1186/1478-811X-832. PubMed PMID: 21182795; PubMed Central PMCID: PMCPMC3022542. 